\title{
LOSS DETERMINATION METHODOLOGY FOR A PIEZOELECTRIC CERAMIC: NEW PHENOMENOLOGICAL THEORY AND EXPERIMENTAL PROPOSALS
}

\author{
KENJI UCHINO ${ }^{*, \dagger, \ddagger, \S}$, YUAN ZHUANG* and SEYIT O. URAL ${ }^{\dagger}$ \\ *Electrical Engineering Department \\ International Center for Actuators and Transducers \\ The Pennsylvania State University \\ University Park, PA 16802, USA \\ ${ }^{\dagger}$ Materials Science and Engineering \\ International Center for Actuators and Transducers \\ The Pennsylvania State University \\ University Park, PA 16802, USA \\ $\ddagger$ Office of Naval Research Global - Asia \\ Minato-ku, Tokyo 106-0032, Japan \\ §kenjiuchino@psu.edu,kenji.uchino@onrg.navy.mil
}

Received 2 December 2010

\begin{abstract}
The key factor to the miniaturization of piezoelectric devices is power density, which is limited by the heat generation or loss mechanisms. There are three loss components in general in piezoelectric vibrators/resonators, i.e., dielectric, elastic and piezoelectric losses. The mechanical quality factor, determined by these three factors, is the Figure Of Merit (FOM) in the sense of loss or heat generation. In this paper, we introduce a new loss phenomenology and innovative measuring methods based on the theory. First, quality factors at resonance and antiresonance for the $k_{31}, k_{33}, k_{t}$ and $k_{15}$ vibration modes are derived theoretically, and the methodology for determining loss factors in various orientations (i.e., loss anisotropy) is provided. For simplicity, we focus on materials with $\infty \mathrm{mm}$ (equivalent to $6 \mathrm{~mm}$ ) crystal symmetry for deriving the loss factors of a polycrystalline ceramic, and 14 different loss factors among 20 in total can be obtained from the measurements. Second, we propose the experimental methods for measuring both mechanical quality factors $Q_{A}$ and $Q_{B}$ at the resonance and antiresonance modes: a continuous admittance/impedance spectrum measuring method (traditional with temperature rise) and a burst mode (to circumvent the temperature effect).
\end{abstract}

Keywords: Piezoelectric; loss factor; quality factor; high power; piezoelectric loss; dielectric loss; elastic loss; admittance/impedance spectrum; burst mode.

${ }^{\S}$ Corresponding author. 


\section{Introduction}

Piezoelectric components gain increasing interest for device miniaturization. Since piezoelectric ultrasonic motors and transformers demonstrated 1/10th size for equivalent-power-level electromagnetic devices with no electromagnetic noise, ${ }^{1,2}$ piezoelectric components have been replacing electromagnetic conjugates. The interest of the industry in ever higher efficiency to achieve a sustainable society while scaling sizes down, forced research towards more power dense piezoelectric technologies. However, the power density in piezoelectric devices is still limited by material's inherent losses which stem from the microscopic domain dynamics, resulting in heat generation. Therefore, to advance device miniaturization it is necessary to clarify the loss mechanisms.

With increasing the electric field and power level, piezoelectrics tend to reveal significant "nonlinearity" in a wide meaning. The non-linear phenomena are composed of (1) a narrow-meaning of non-linearity in dielectric, elastic and piezoelectric constants in terms of electric field and stress and (2) hysteresis (or time-lag) of the polarization and strain versus electric field and stress. Regarding the non-linearity of the physical parameters, various analytical models have been proposed so far, which explained the jump phenomenon and hysteresis in the admittance/impedance spectrum during increasing and decreasing the driving frequency. ${ }^{3-5}$ However, there seems to be a lack of effort on the hysteresis phenomenon, which is directly related with loss and heat generation.

Losses in piezoelectrics are considered to have three components: dielectric, elastic and piezoelectric. ${ }^{6}$ The dielectric and elastic loss factors are commonly reported by researchers and in manufacturer's catalogues, while little attention has so far been paid to the "piezoelectric loss" factor. ${ }^{7}$ However, relatively large piezoelectric loss factors were reported in our previous study, which also explained the discrepancy between the conventional theoretical expectation and experimental result of mechanical quality factors. ${ }^{8-10}$ We further classified each loss into intensive and extensive components, which are briefly summarized in the next section. ${ }^{11}$

Mechanical quality factors play a significant role in the study of losses in piezoelectrics. A higher mechanical quality factor increases the efficiency and reduces heat generation. The quality factor is basically related to dielectric, elastic and piezoelectric loss factors. Besides, a higher quality factor at the antiresonance is usually observed in experiments, in comparison with that at the resonance. $^{12,13}$ Due to the misconception in the conventional IEEE Standard on piezoelectrics ${ }^{14}$ that "the mechanical quality factors for resonance and antiresonance are the same", not many researchers have argued on this issue, or the "piezoelectric loss" has continuously been neglected in these 30 years.

In this paper, we provide comprehensive derivations of piezoelectric quality factors obtained from phenomenological treatment for both resonance and antiresonance in four vibration modes: $k_{31}, k_{33}, k_{t}$ and $k_{15}$ modes, and a systematical methodology to determine the loss factors in different crystal orientations is then proposed based on the quality factor formulas. Further, an innovative experimental measuring system (HiPoCS - High Power piezoelectric Characterization System) is introduced in order to measure both mechanical quality factors $Q_{A}$ and $Q_{B}$ at the resonance and antiresonance modes: a continuous admittance/impedance measurement method (traditional with temperature rise) and a burst mode (to avoid the temperature effect). Note that we considered only the hysteresis in physical parameters, which is directly relevant to loss, heat generation, and efficiency of piezoelectric vibrators/resonators, and neglected the narrowmeaning non-linearity in this paper.

\section{Piezoelectric Losses and Quality Factor Definitions}

Complex parameters are integrated to express the small hysteresis losses in piezoelectrics. ${ }^{15}$ We use $\tan \delta^{\prime}, \tan \phi^{\prime}$ and $\tan \theta^{\prime}$ to represent "intensive" dielectric, elastic and piezoelectric loss factors, respectively. The "extensive" loss factors are given by corresponding notations without prime. ${ }^{11}$

$$
\begin{aligned}
\varepsilon^{X^{*}} & =\varepsilon^{X}\left(1-j \tan \delta^{\prime}\right), \\
s^{E^{*}} & =s^{E}\left(1-j \tan \phi^{\prime}\right), \\
d^{*} & =d\left(1-j \tan \theta^{\prime}\right), \\
\kappa^{x^{*}} & =\kappa^{x}(1+j \tan \delta), \\
c^{D^{*}} & =c^{D}(1+j \tan \phi), \\
h^{*} & =h(1+j \tan \theta) .
\end{aligned}
$$


Here $j$ is the imaginary notation, $\varepsilon^{X}$ the dielectric constant under constant stress, $\kappa^{x}$ the inverse dielectric constant under constant strain, $s^{E}$ the elastic compliance under constant electric field, $c^{D}$ the elastic stiffness under constant electric displacement, $d$ the piezoelectric constant and $h$ the inverse piezoelectric charge constant. For piezoelectric ceramics,

$$
\varepsilon^{x^{*}}=\varepsilon^{x}(1-j \tan \delta) .
$$

It is notable to describe here that there is limitation in physics of this complex parameter usage: the value of the dissipation factor or loss tangent should be very small (typically less than 1\%) to keep the equivalency to the combination treatment of the dissipation function theory and thermodynamics. ${ }^{16}$

Figure 1 shows schematic illustrations for various hysteresis curves: (a) electric field $E$ versus electric displacement $D$ (or polarization $P$ ), (b) stress $X$ versus strain $x$, (c) $E$ versus $x$ and (d) $X$ versus $D$. The energy and loss can be calculated based on the hysteresis area. ${ }^{11}$ We reported the piezoelectric loss determined from the pseudo-static measurements using a hydraulic pressure apparatus in the past. ${ }^{8,17}$ However, this conventional method with a mechanical stress apparatus is difficult for inexperienced engineers to conduct, and not many researchers have utilized this methodology.

Because of this background, we propose here a simple, user-friendly methodology for obtaining three

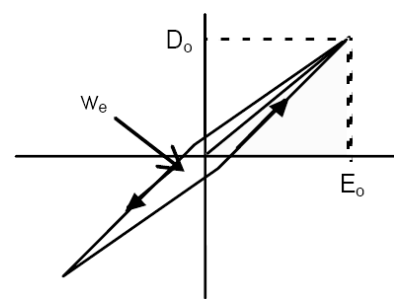

(a)

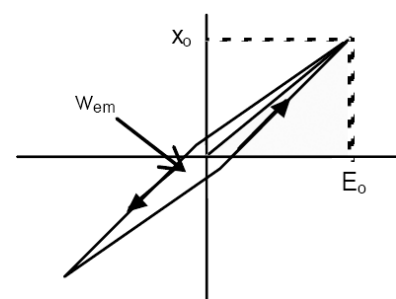

(c)



(b)



(d)
Fig. 1. Schematic representation of experimentally determined hysteresis curves: (a) $D-E$ (stress-free), (b) $x-X$ (shortcircuit), (c) $x-E$ (stress-free) and (d) $D-X$ (short-circuit).

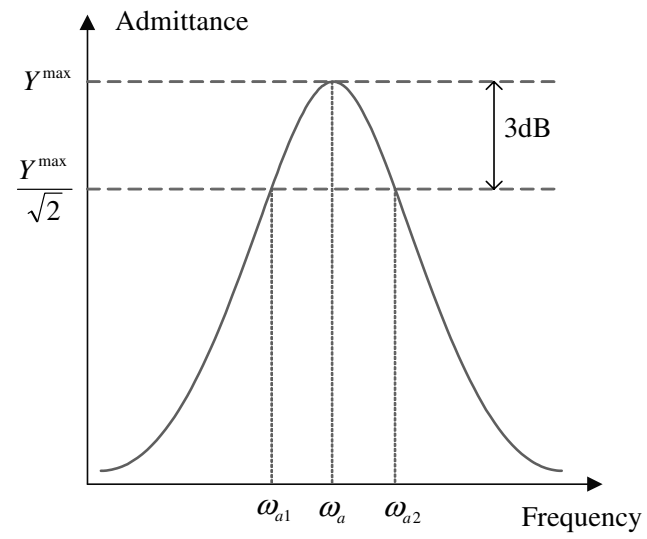

(a)



(b)

Fig. 2. Definitions of quality factors at resonance (a) and antiresonance (b).

losses separately from the admittance/impedance characterizations. Loss factors are closely related to the mechanical quality factors, the Figure Of Merit (FOM) of a material in terms of loss and heat generation, at both resonance and antiresonance modes. Figure 2 illustrates schematically the definitions of the mechanical quality factors at resonance and antiresonance. $Q_{A}$ and $Q_{B}$ are used to represent the quality factors under the resonance ( $A$-type resonance) and antiresonance (B-type resonance), respectively,

$$
\begin{aligned}
Q_{A} & =\frac{\omega_{a}}{\omega_{a 2}-\omega_{a 1}}, \\
Q_{B} & =\frac{\omega_{b}}{\omega_{b 2}-\omega_{b 1}},
\end{aligned}
$$

where $\omega_{a}$ is the resonance frequency, $\omega_{b}$ is the antiresonance frequency, and $\left(\omega_{a 2}-\omega_{a 1}\right)$ and $\left(\omega_{b 2}-\omega_{b 1}\right)$ correspond to the $3 \mathrm{~dB}$ bandwidth in the admittance 
curves around the resonance and antiresonance peaks, respectively.

\section{Derivation of Quality Factors}

We derived the mechanical quality factors for piezoelectric samples in $k_{31}, k_{33}, k_{t}$ and $k_{15}$ vibration modes. All the quality factor expressions are derived from the impedance expressions of different modes utilizing first order approximation. Since the derivation details are skipped here, refer to our previous papers. $^{18,19}$

\section{1. $k_{31}$ mode}

The configuration of a $k_{31}$ sample is shown in Fig. 3, and the results of quality factors are given by ${ }^{18}$

$$
\begin{gathered}
Q_{A, 31}=\frac{1}{\tan \phi_{11}^{\prime}}, \\
\frac{1}{Q_{B, 31}}=\frac{1}{Q_{A, 31}}-\frac{2}{1+\left(\frac{1}{k_{31}}-k_{31}\right)^{2} \Omega_{B, 31}^{2}} \\
\cdot\left(2 \tan \theta_{31}^{\prime}-\tan \delta_{33}^{\prime}-\tan \phi_{11}^{\prime}\right) .
\end{gathered}
$$

Here $\tan \delta_{33}^{\prime}, \tan \phi_{11}^{\prime}, \tan \theta_{31}^{\prime}$ are loss factors for $\varepsilon_{33}^{X}, s_{11}^{E}, d_{31}$, respectively. The parameter $\Omega_{B, 31}$ is proportional to the antiresonance frequency,

$$
\Omega_{B, 31}=\frac{\omega_{b} L}{2 v_{11}^{E}},
$$

where $v_{11}^{E}$ is the sound velocity of the material in the direction of length under constant $E$.

\section{2. $k_{33}$ mode}

The configuration of a $k_{33}$ sample is shown in Fig. 4, and the results of quality factors are given by

$$
\begin{aligned}
\frac{1}{Q_{B, 33}}= & \frac{1}{1-k_{33}^{2}} \\
& \cdot\left[\tan \phi_{33}^{\prime}-k_{33}^{2}\left(2 \tan \theta_{33}^{\prime}-\tan \delta_{33}^{\prime}\right)\right],
\end{aligned}
$$

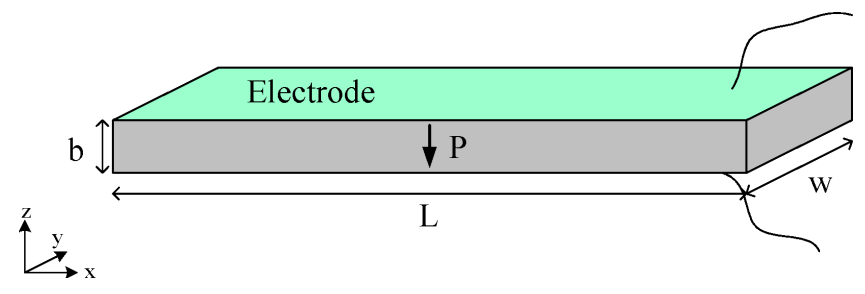

Fig. 3. Configuration of a $k_{31}$ sample.

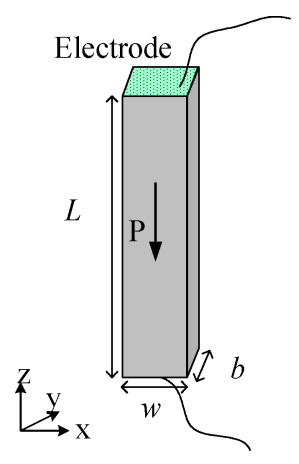

Fig. 4. Configuration of a $k_{33}$ sample.

$$
\begin{aligned}
\frac{1}{Q_{A, 33}}= & \frac{1}{Q_{B, 33}}+\frac{2}{k_{33}^{2}-1+\Omega_{A, 33}^{2} / k_{33}^{2}} \\
& \cdot\left(2 \tan \theta_{33}^{\prime}-\tan \delta_{33}^{\prime}-\tan \phi_{33}^{\prime}\right) .
\end{aligned}
$$

Here $\tan \delta_{33}^{\prime}, \tan \phi_{33}^{\prime}, \tan \theta_{33}^{\prime}$ are loss factors for $\varepsilon_{33}^{X}, s_{33}^{E}, d_{33}$, respectively. The parameter $\Omega_{A, 33}$ is proportional to the resonance frequency,

$$
\Omega_{A, 33}=\frac{\omega_{a} L}{2 v_{33}^{D}},
$$

where $v_{33}^{D}$ is the sound velocity of the material in the direction of length under constant $D$.

\section{3. $k_{t}$ mode}

The configuration of a $k_{t}$ sample is shown in Fig. 5, and the results of quality factors are given by

$$
\begin{gathered}
Q_{B, t}=\frac{1}{\tan \phi_{33}}, \\
\frac{1}{Q_{A, t}}=\frac{1}{Q_{B, t}}+\frac{2}{k_{t}^{2}-1+\Omega_{A, t}^{2} / k_{t}^{2}} \\
\cdot\left(\tan \delta_{33}+\tan \phi_{33}-2 \tan \theta_{33}\right) .
\end{gathered}
$$

Here $\tan \delta_{33}, \tan \phi_{33}, \tan \theta_{33}$ are loss factors for $\varepsilon_{33}^{x}, c_{33}^{D}, h_{33}$, respectively. The parameter $\Omega_{A, t}$ is proportional to the resonance frequency,

$$
\Omega_{A, t}=\frac{\omega_{a} b}{2 v_{t}^{D}},
$$



Fig. 5. Configuration of a $k_{t}$ sample. 
where $v_{t}^{D}$ is the sound velocity of the material in the direction of thickness under constant $D$.

\section{4. $k_{15}$ mode}

There are two configurations of $k_{15}$ samples, as shown in Fig. 6. In Fig. 6(a) $L \gg t$, and this thickness shear mode corresponds to the constant induction condition. In Fig. 6(b) $L \ll t$, which leads to the length shear mode with constant electric field condition.

The quality factors in constant induction condition are given by

$$
\begin{gathered}
Q_{B, 15}^{D}=\frac{1}{\tan \phi_{55}}, \\
\frac{1}{Q_{A, 15}^{D}}=\frac{1}{Q_{B, 15}^{D}}+\frac{2}{k_{15}^{2}-1+\Omega_{A, 15}^{2} / k_{15}^{2}} \\
\cdot\left(\tan \delta_{11}+\tan \phi_{55}-2 \tan \theta_{15}\right) .
\end{gathered}
$$

Here $\tan \delta_{11}, \tan \phi_{55}, \tan \theta_{15}$ are loss factors for $\varepsilon_{11}^{x}, c_{55}^{D}, h_{15}$, respectively. The parameter $\Omega_{A, 15}$ is proportional to the resonance frequency,

$$
\Omega_{A, 15}=\frac{\omega_{a} t}{2 v_{55}^{D}},
$$

where $v_{55}^{D}$ is the sound velocity under constant $D$.

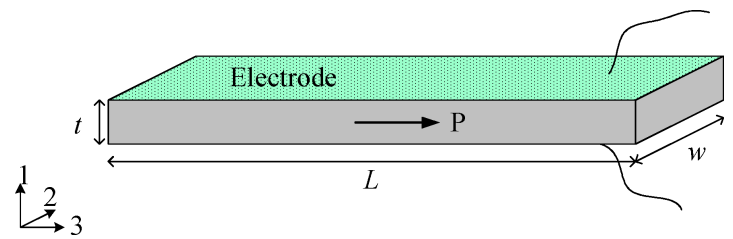

(a)

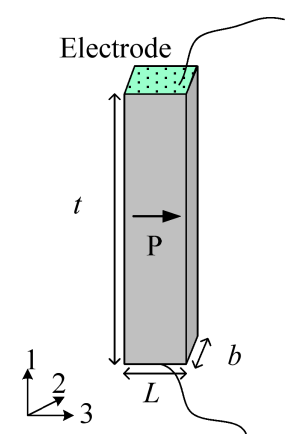

(b)

Fig. 6. Configuration of $k_{15}$ samples: (a) constant induction condition; (b) constant field condition.
On the other hand, the quality factors in constant field condition are given by

$$
\begin{gathered}
Q_{A, 15}^{E}=\frac{1}{\tan \phi_{55}^{\prime}}, \\
\frac{1}{Q_{B, 15}^{E}}=\frac{1}{Q_{A, 15}^{E}}-\frac{2}{1+\left(\frac{1}{k_{15}}-k_{15}\right)^{2} \Omega_{B, 15}^{2}} \\
\cdot\left(2 \tan \theta_{15}^{\prime}-\tan \delta_{11}^{\prime}-\tan \phi_{55}^{\prime}\right) .
\end{gathered}
$$

Here $\tan \delta_{11}^{\prime}, \tan \phi_{55}^{\prime}, \tan \theta_{15}^{\prime}$ are loss factors for $\varepsilon_{11}^{X}, s_{55}^{E}, d_{15}$, respectively. The parameter $\Omega_{B, 15}$ is proportional to the antiresonance frequency,

$$
\Omega_{B, 15}=\frac{\omega_{b} L}{2 v_{55}^{E}},
$$

where $v_{55}^{E}$ is the sound velocity under constant $E$.

\section{Interrelationship Between Intensive and Extensive Loss Factors}

The intensive and extensive loss factors are mutually inter-related. Let us discuss this issue based on the simplest 1-D model here. The electromechanical coupling coefficient is given by

$$
k^{2}=\frac{d^{2}}{s^{E}\left(\varepsilon^{X} \varepsilon_{0}\right)}=\frac{h^{2}}{c^{D}\left(\kappa^{x} / \varepsilon_{0}\right)} .
$$

Taking into account

$$
\begin{gathered}
\varepsilon^{x}=\varepsilon^{X}\left(1-k^{2}\right), \\
s^{D}=s^{E}\left(1-k^{2}\right),
\end{gathered}
$$

the intensive and extensive loss factors are verified to keep the following relationship:

$$
\begin{gathered}
{\left[\begin{array}{c}
\tan \delta^{\prime} \\
\tan \phi^{\prime} \\
\tan \theta^{\prime}
\end{array}\right]=K\left[\begin{array}{c}
\tan \delta \\
\tan \phi \\
\tan \theta
\end{array}\right],} \\
K=\frac{1}{1-k^{2}}\left[\begin{array}{ccc}
1 & k^{2} & -2 k^{2} \\
k^{2} & 1 & -2 k^{2} \\
1 & 1 & -1-k^{2}
\end{array}\right] .
\end{gathered}
$$

Here, the matrix $K$ is proven to be involutory; i.e., $K^{2}=I$, or $K=K^{-1}$, where $I$ is the identity 
matrix. Hence the conversion relationship between the intensive (prime) and extensive (non-prime) exhibits full symmetry. Since we are to utilize inverse matrices in the $3-\mathrm{D}$ case, the discussion becomes more complicated than the above simplest $1-D$ case. In the case of matrix calculations for piezoelectric ceramics, Eqs. (25) and (28) only hold for $k_{15}$ mode.

\section{Methodology to Determine Loss Factors}

In this section, we provide a systematical methodology to determine different loss factors for piezoelectric ceramics with $\infty \mathrm{mm} / 6 \mathrm{~mm}$ crystal symmetry. There are 20 parameters for such materials: $\varepsilon_{33}^{X}, \varepsilon_{11}^{X}, \varepsilon_{33}^{x}, \varepsilon_{11}^{x} ; s_{11}^{E}, s_{12}^{E}, s_{13}^{E}, s_{33}^{E}, s_{55}^{E}, c_{11}^{D}$, $c_{12}^{D}, c_{13}^{D}, c_{33}^{D}, c_{55}^{D} ; d_{31}, d_{33}, d_{15}, h_{31}, h_{33}, h_{15}$. By the characterizations of $k_{13}, k_{33}, k_{t}$ and $k_{15}$ modes, 14 loss factors can be obtained. The procedures are listed as follows.

(i) Measure the resonance and antiresonance frequencies to derive the values of $k_{31}, k_{33}, k_{t}$ and $k_{15} \cdot{ }^{14}$

$$
\begin{aligned}
\frac{k_{31}^{2}}{1-k_{31}^{2}} & =\frac{\pi}{2} \frac{\omega_{b, 31}}{\omega_{a, 31}} \tan \left[\frac{\pi\left(\omega_{b, 31}-\omega_{a, 31}\right)}{2 \omega_{a, 31}}\right], \\
k_{33}^{2} & =\frac{\pi}{2} \frac{\omega_{a, 33}}{\omega_{b, 33}} \tan \left[\frac{\pi\left(\omega_{b, 33}-\omega_{a, 33}\right)}{2 \omega_{b, 33}}\right], \\
k_{t}^{2} & =\frac{\pi}{2} \frac{\omega_{a, t}}{\omega_{b, t}} \tan \left[\frac{\pi\left(\omega_{b, t}-\omega_{a, t}\right)}{2 \omega_{b, t}}\right], \\
k_{15}^{2} & =\frac{\pi}{2} \frac{\omega_{a, 15}}{\omega_{b, 15}} \tan \left[\frac{\pi\left(\omega_{b, 15}-\omega_{a, 15}\right)}{2 \omega_{b, 15}}\right] .
\end{aligned}
$$

Equation (33) should be applied for the thickness shear mode $(L \gg t)$. The result of $k_{15}$ is same as the one for the length shear mode. ${ }^{20}$

(ii) Using an LCR meter to characterize dielectric losses: Measure the capacitance of $k_{31}$ mode to obtain $\tan \delta_{33}^{\prime}$ (low frequency below the resonance) and $\tan \delta_{33}$ (high frequency above the resonance/antiresonance). Measure the capacitance of the thickness shear mode to obtain $\tan \delta_{11}^{\prime}$ (low frequency) and $\tan \delta_{11}$ (high frequency). ${ }^{14}$ (iii) Measure $Q_{A}$ in $k_{31}$ mode to get $\tan \phi_{11}^{\prime}$, the loss factor for $s_{11}^{E}$.

$$
\tan \phi_{11}^{\prime}=\frac{1}{Q_{A, 31}} .
$$

(iv) Measure $Q_{B}$ in $k_{31}$ mode, and then calculate $\tan \theta_{31}^{\prime}$, which is the loss factor for $d_{31}$.

$$
\begin{aligned}
\tan \theta_{31}^{\prime}= & \frac{\tan \delta_{33}^{\prime}+\tan \phi_{11}^{\prime}}{2} \\
& +\frac{1}{4}\left(\frac{1}{Q_{A, 31}}-\frac{1}{Q_{B, 31}}\right) \\
& \cdot\left[1+\left(\frac{1}{k_{31}}-k_{31}\right)^{2} \Omega_{B, 31}^{2}\right] .
\end{aligned}
$$

(v) Measure $Q_{A}$ and $Q_{B}$ in $k_{33}$ mode to derive $\tan \phi_{33}^{\prime}$ for $s_{33}^{E}$, and $\tan \theta_{33}^{\prime}$ for $d_{33}$.

$$
\begin{aligned}
\tan \phi_{33}^{\prime}-2 k_{33}^{2} \tan \theta_{33}^{\prime} \\
=\left(1-k_{33}^{2}\right) \frac{1}{Q_{B, 33}}-k_{33}^{2} \tan \delta_{33}^{\prime} . \\
\tan \phi_{33}^{\prime}-2 \tan \theta_{33}^{\prime} \\
=\left(\frac{1}{Q_{B, 33}}-\frac{1}{Q_{A, 33}}\right) \\
\cdot \frac{k_{33}^{2}-1+\Omega_{A, 33}^{2} / k_{33}^{2}}{2}-\tan \delta_{33}^{\prime} .
\end{aligned}
$$

(vi) Measure $Q_{B}$ in $k_{t}$ mode to get $\tan \phi_{33}$ for $c_{33}^{D}$.

$$
\tan \phi_{33}=\frac{1}{Q_{B, t}} \text {. }
$$

(vii) Measure $Q_{A}$ in $k_{t}$ mode to obtain $\tan \theta_{33}$ for $h_{33}$.

$$
\begin{aligned}
\tan \theta_{33}= & \frac{\tan \delta_{33}+\tan \phi_{33}}{2} \\
& +\frac{1}{4}\left(\frac{1}{Q_{B, t}}-\frac{1}{Q_{A, t}}\right) \\
& \cdot\left(k_{t}^{2}-1+\Omega_{A, t}^{2} / k_{t}^{2}\right) .
\end{aligned}
$$

(viii) Measure $Q_{A}$ in $k_{15}$ mode under constant $E$ condition to get $\tan \phi_{55}^{\prime}$, the loss factor for $s_{55}^{E}$.

$$
\tan \phi_{55}^{\prime}=\frac{1}{Q_{A, 15}^{E}} .
$$


(ix) Measure $Q_{B}$ under constant $E$ condition, and then calculate $\tan \theta_{15}^{\prime}$, the loss factor for $d_{15}$.

$$
\begin{aligned}
\tan \theta_{15}^{\prime}= & \frac{\tan \delta_{11}^{\prime}+\tan \phi_{55}^{\prime}}{2} \\
& +\frac{1}{4}\left(\frac{1}{Q_{A, 15}^{E}}-\frac{1}{Q_{B, 15}^{E}}\right) \\
& \cdot\left[1+\left(\frac{1}{k_{15}}-k_{15}\right)^{2} \Omega_{B, 15}^{2}\right] .
\end{aligned}
$$

(x) Measure $Q_{B}$ in $k_{15}$ mode under constant $D$ condition to get $\tan \phi_{55}$ for $c_{55}^{D}$.

$$
\tan \phi_{55}=\frac{1}{Q_{B, 15}^{D}}
$$

(xi) Measure $Q_{A}$ under constant $D$ condition, and derive $\tan \theta_{15}$ for $h_{15}$.

$$
\begin{aligned}
\tan \theta_{15}= & \frac{\tan \delta_{11}+\tan \phi_{55}}{2} \\
& +\frac{1}{4}\left(\frac{1}{Q_{B, 15}^{D}}-\frac{1}{Q_{A, 15}^{D}}\right) \\
& \cdot\left(k_{15}^{2}-1+\Omega_{A, 15}^{2} / k_{15}^{2}\right) .
\end{aligned}
$$

We have described how the loss factors of piezoelectrics are derived from the $k_{31}, k_{33}, k_{t}$ and $k_{15}$ resonator samples. 14 different loss factors can be determined: $\delta_{11}^{\prime}, \delta_{11}^{\prime}, \delta_{33}^{\prime}, \delta_{33} ; \phi_{11}^{\prime}, \phi_{33}^{\prime}, \phi_{33}, \phi_{55}^{\prime}, \phi_{55}$; $\theta_{31}^{\prime}, \theta_{33}^{\prime}, \theta_{33}, \theta_{15}^{\prime}, \theta_{15}$.

In order to determine the remaining 6 loss factors, we need to characterize $k_{p}$ mode and make further derivations.

\section{Mechanical Quality Factor Measuring Techniques}

We are proposing a new method on how to determine three losses (dielectric, elastic and piezoelectric) for each mode based on three measurement values: (1) $Q_{A}$ value at the resonance mode, (2) $Q_{B}$ value at the antiresonance mode and (3) the dielectric loss at off-resonance $\left(\tan \delta^{\prime}\right.$ or $\tan \delta$ at a low or high frequency). These three experimental values can determine all three (dielectric, elastic and piezoelectric) losses, in general. We will review here our experimental efforts on the determination of high power piezoelectric performances, in particular, mechanical quality factor $Q_{M}$ through our HiPoCS (High Power piezoelectric Characterization System) history.

\subsection{HiPoCS Version I (Resonance under constant voltage, 1980s)}

Because commercially-available "Impedance Analyzers" at that time did not generate high voltage/ current for measuring the high power piezoelectric performance, we commercialized "Frequency Response Analyzer" (500 V, 20 A, 1 MHz maximum) from NF Corporation, Japan. ${ }^{3}$ We reported the existence of the critical threshold voltage and the maximum vibration velocity for a piezoelectric, above which the piezoelectric drastically increases the heat generation and becomes a ceramic heater. However, HiPoCS Version I did not have the capability to measure the piezoelectric loss. This NF high voltage/current power supply is still a key device in the present Version V.

\subsection{HiPoCS Version II (Quasi-static measurement, 1990s)}

We determined dissipation factors, $\tan \delta^{\prime}, \tan \phi^{\prime}$, and $\tan \theta^{\prime}$, separately from (a) $D$ versus $E$, (b) $x$ versus $X$, (c) $x$ versus $E$ and (d) $D$ versus $X$ curves (Fig. 1) for a soft PZT based multilayer actuator. ${ }^{8}$ Using a jig for applying stress, we measured the $x$ versus $X$ and $D$ versus $X$ relationships. ${ }^{8}$ This is the first paper which reported that the piezoelectric loss $\tan \theta^{\prime}$ is not negligibly small as believed by the previous researchers, but rather large; comparable to the dielectric and elastic losses: $\tan \theta^{\prime}(=0.08)>$ $(1 / 2)\left[\tan \delta^{\prime}(=0.06)+\tan \phi^{\prime}(=0.08)\right]$. Though HiPoCS Version II has the capability to measure three losses separately, this equipment has not become popular, because it was bulky and measuring the mechanical load performance was very timeconsuming.

\subsection{HiPoCS Version III (Pulse drive, 1990s)}

In order to establish a simple and quick characterization method, we introduced an alternative method, i.e., the pulse drive method for measuring high voltage piezoelectric characteristics. By applying a step electric field to a piezoelectric 
sample, the transient vibration displacement corresponding to the desired mode (extensional, bending, etc.) was measured. The resonance period, stabilized displacement and damping constant were obtained experimentally, from which the elastic compliance, piezoelectric constant, mechanical quality factor and electromechanical coupling factor can be calculated. ${ }^{16,17}$

This HiPoCS Version III was attractive especially for its simple setup and low cost. However, drawbacks included: (1) only elastic loss or mechanical quality factor $Q_{M}$ at resonance can be measured, (2) the accuracy is not high.

\subsection{HiPoCS Version IV (Resonance under constant current, 1990s)}

In the end of $1990 \mathrm{~s}$, we re-started the resonance method, but this time under a constant current condition, as opposed to the traditional constant voltage method. ${ }^{12,17}$ Though the traditional constant voltage measurement technique is simple, it is inherently flawed as it introduces a significant distortion of the admittance frequency spectrum when the sample is driven by increasing voltage values, due to the non-linear behavior of elastic compliance at high vibration amplitude. ${ }^{3,4}$ Figure 7 (a) exemplifies the problem, where the admittance spectrum is skewed towards lower frequencies, with a jump around the maximum admittance point. Since the vibration amplitude is significantly amplified as the resonance frequency is approached under a constant voltage drive, the nonlinear elastic property exhibits this sort of abnormal phenomenon. Thus, neither the resonance frequency nor the mechanical quality factor can be determined precisely from these skewed spectra.

In order to avoid this problem with a constant voltage measurement, HiPoCS Version IV adopted a constant current measurement technique. ${ }^{17}$ Since the vibration amplitude is primarily proportional to the driving current (not the voltage) at the resonance, a constant current condition guarantees almost constant vibration amplitude through the resonance frequency region, avoiding the spectrum distortion due to the elastic non-linearity. As demonstrated in Fig. 7(b), the spectra exhibit symmetric curves, from which the resonance frequency and the mechanical quality factor $Q_{m}$ can be determined precisely. The mechanical quality factor $Q_{m}$ or loss factor $\tan \phi^{\prime}$ is obtained from $Q_{m}=\omega_{R} / 2 \Delta \omega$, where $2 \Delta \omega$ is a full width of the $1 / \sqrt{2}$ of the maximum admittance value at $\omega_{R}$. HiPoCS Version IV improved the capability for measuring the mechanical quality factor $Q_{m}$ at the resonance region, but did not provide information on the piezoelectric loss $\tan \theta^{\prime}$.

In order to identify a full set of high power electromechanical coupling parameters and the loss factors of a piezoelectric, both resonance and antiresonance vibration performance (i.e., $Q_{A}$ and $Q_{B}$ ) should be precisely measured simultaneously. Note that identification of the antiresonance $Q_{B}$ requires primarily the constant voltage method.

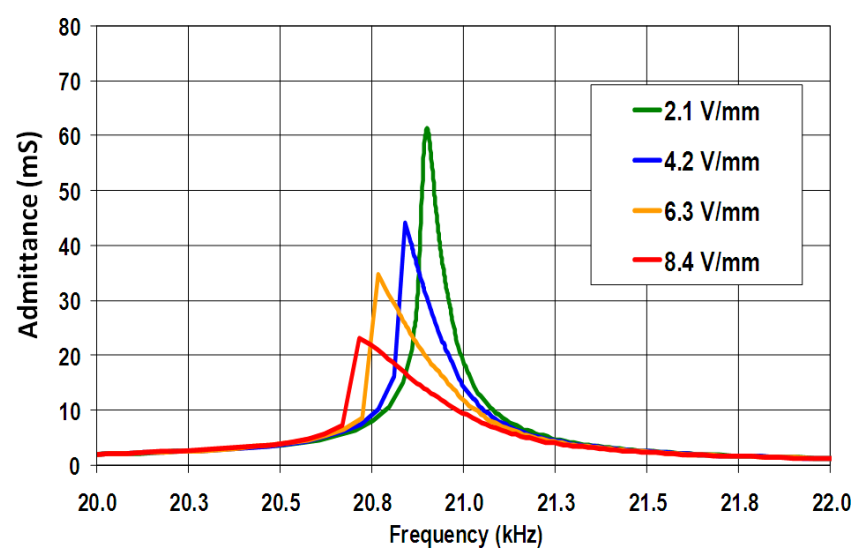

(a)



(b)

Fig. 7. (Color online) Experimentally obtained admittance frequency spectra under (a) constant voltage and (b) constant current condition. Note the skew-distorted spectrum with a jump under a constant voltage condition. 


\subsection{HiPoCS Version V (Resonance under constant vibration velocity, 2000s)}

In order to obtain both mechanical quality factors $Q_{A}$ and $Q_{B}$ precisely for adopting the above mentioned user-friendly methodology (Sec. 5), both resonance and antiresonance vibration performance should be measured simultaneously without changing the experimental equipment. As already discussed, basically, $Q_{A}$ can be determined by the constant current method around the resonance (A-type), while $Q_{B}$ should be determined by the constant voltage method around the antiresonance (B-type). Thus, we developed HiPoCS Version V illustrated in Fig. 8, which is capable of measuring the impedance/admittance curves by keeping the following various conditions: (1) constant voltage, (2) constant current, (3) constant vibration velocity of a piezoelectric sample and (4) constant input power. ${ }^{21}$ In addition, the system is equipped with an infrared image sensor to monitor the heat generation and the temperature distributed in the test sample. We demonstrated the usefulness of the new system in a rectangular piezoelectric plate in the whole frequency range including the resonance and antiresonance frequencies.

An amplitude controlled sinusoidal signal is produced by a function generator (HP 33120A) via an amplifier (NF 4010, NF Electric Instruments). The sample current is detected by a clamp-on AC current sensor (TCP 305, Tektronix). The voltage, current and displacement waveforms of the PZT plates/disks are monitored by two digital oscilloscopes (TDS 3014B, Tektronix) and logged by the $\mathrm{PC}$. The temperature on the surface of the samples nodal point is measured by an infrared spot thermometer (HIOKI 3445, HIOKI, E.E., Corp.) and thermal images are taken with a thermal camera (ThermaCAM A40 FLIR Systems outfitted with $200 \mu \mathrm{m}$ Lens). Vibration amplitude on the edge of the piezoelectric plate is measured with a laser interferometer (Polytec OFV 511). The sample used for evaluation was a $80 \times 11 \times 2 \mathrm{~mm}^{3}$ plate. The plate sample was supported via two pins at the nodal point (center) and driven at the first extensional $k_{31}$ resonance frequency.

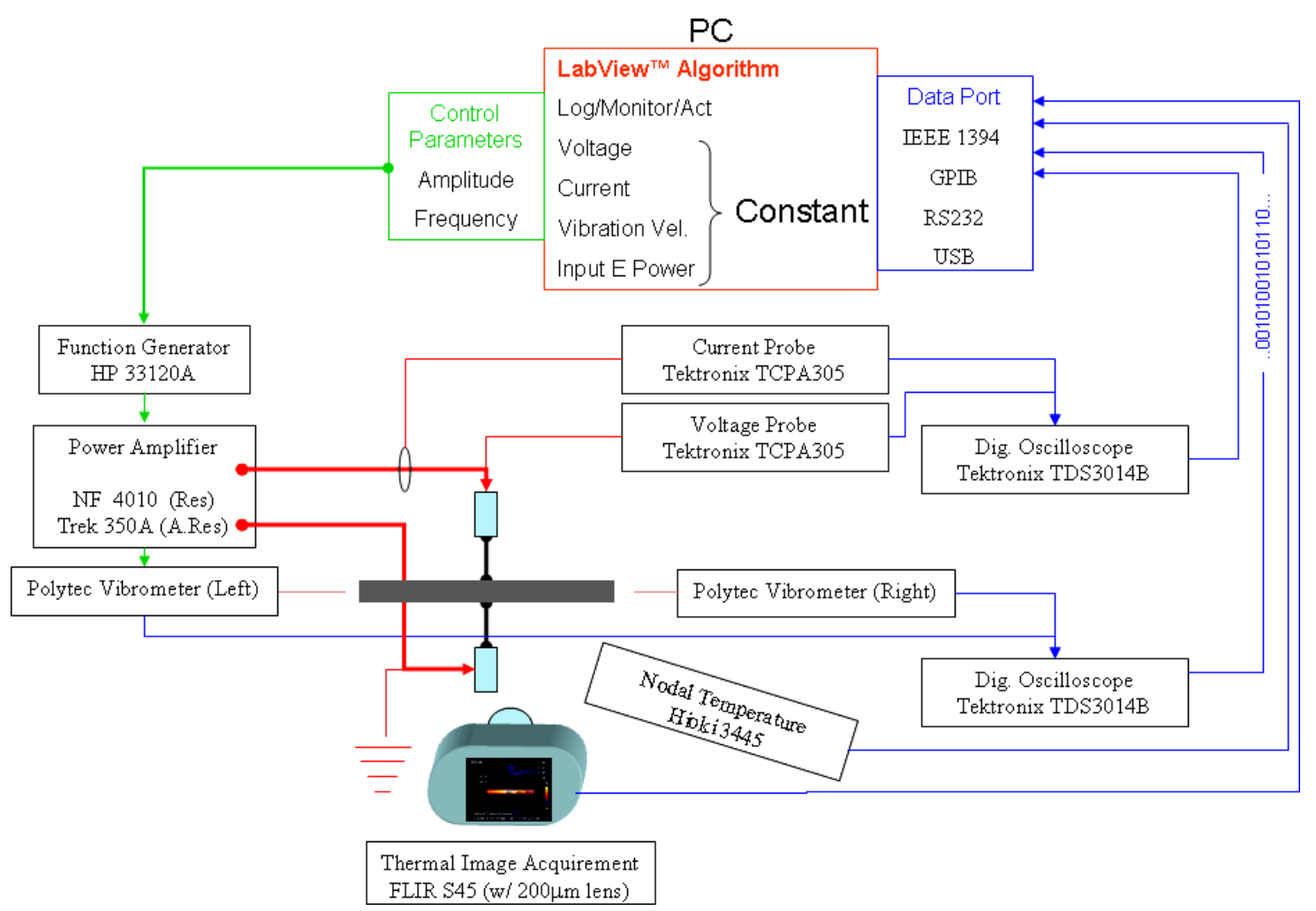

Fig. 8. (Color online) HiPoCS Version V. 


\subsubsection{Mechanical quality factors at resonance and antiresonance}

The resonance and antiresonance are both electromechanical resonances, and both generate large displacement amplitudes, which can be used for actuator/transducer applications. The major difference is the driving electrical admittance/ impedance; low voltage \& high current drive versus high voltage $\&$ low current drive. Another difference can be found in their mechanical quality factor $Q_{m}$; in most of the PZT materials, $Q_{B}$ is higher than $Q_{A}$, in other words the antiresonance mode has a higher efficiency, which we firstly pointed out.

Table 1 shows two examples of the electromechanical parameters (in particular $Q_{A}$ and $Q_{B}$ ). In the experiment, a hard PZT based ceramic sample APC 841 (APC International) and a manganesedoped $\mathrm{Pb}\left(\mathrm{Mg}_{1 / 3} \mathrm{Nb}_{2 / 3}\right) \mathrm{O}_{3}-\mathrm{PbTiO}_{3}$ (Mn-PMN-PT) single crystal were used with a $k_{31}$ configuration. By applying Eq. (11), the piezoelectric loss factor $\tan \theta^{\prime}$ was obtained. We reported that the piezoelectric loss is comparable to, and even larger than other two losses (dielectric and elastic). ${ }^{22}$

The difference between $Q_{B}$ and $Q_{A}$ is mainly determined by the term $\left(\tan \delta^{\prime}+\tan \phi^{\prime}-2 \tan \theta^{\prime}\right)$. According to Eq. (11), if $2 \tan \theta^{\prime}>\left(\tan \delta^{\prime}+\tan \phi^{\prime}\right)$, then $Q_{B}>Q_{A}$, which is consistent with the above experimental observations. Otherwise, if $2 \tan \theta^{\prime}<$ $\left(\tan \delta^{\prime} \tan \phi^{\prime}\right.$ ), then $Q_{A}>Q_{B}$ which has not been experimentally observed so far. The condition $2 \tan \theta^{\prime}=\left(\tan \delta^{\prime}+\tan \phi^{\prime} \quad\right.$ sets $Q_{B}=Q_{A}$, which corresponds to the assumption of the IEEE Standard. The argument here is in exact agreement with the results in Sec. 6.2, which were obtained by a quasi-static hysteresis measurement method.

\subsubsection{Vibration velocity dependence of losses}

Figure 9 shows the vibration velocity dependence of the mechanical quality factors $Q_{A}$ and $Q_{B}$, and corresponding temperature rise for $\mathrm{A}$ (resonance) and $\mathrm{B}$ (antiresonance) type resonances of a longitudinally vibrating PZT ceramic transducer through the



Fig. 9. Vibration velocity dependence of the mechanical quality factors $Q_{A}$ and $Q_{B}$, and corresponding temperature rise for $\mathrm{A}$ (resonance) and $\mathrm{B}$ (antiresonance) type resonances of a longitudinally vibrating PZT ceramic transducer through the transverse piezoelectric effect $d_{31}$.

transverse piezoelectric effect $d_{31}$ (the sample size is inserted). $Q_{m}$ is almost constant for a small electric field/vibration velocity, but above a certain vibration level $Q_{m}$ degrades significantly, where temperature rise starts to be observed too. In order to evaluate the mechanical vibration level, we introduced the vibration velocity at the rectangular plate tip. The maximum vibration velocity is defined at the velocity where a $20^{\circ} \mathrm{C}$ temperature rise at the nodal point from room temperature occurs. Note that even if the driving voltage/field is increased, additional energy will merely converted to heat (i.e., PZT becomes a ceramic heater!) without increasing the vibration amplitude.

When we compare the change trends in $Q_{A}$ and $Q_{B}, Q_{B}$ is higher than $Q_{A}$ in all vibration level. Accordingly, the heat generation in the B-type (antiresonance) mode is superior to the A-type (resonance) mode under the same vibration velocity level (in other words, the maximum vibration velocity is higher for $Q_{B}$ than for $Q_{A}$ ).

\subsubsection{Heat generation at resonance and antiresonance}

Figure 10 plots the frequency spectra of voltage and current (top) and power (bottom) under constant

Table 1. Electromechanical parameters of PZT ceramic APC 841 and Mn-PMN-PT single crystal ( $k_{31}$ mode).

\begin{tabular}{lcccccr}
\hline Material & $Q_{A}\left(1 / \tan \phi^{\prime}\right)$ & $\tan \phi^{\prime}$ & $Q_{B}$ & $\tan \delta^{\prime}$ & $k_{31}$ & $\tan \theta^{\prime}$ \\
\hline APC 841 & 1183 & 0.00085 & 1986 & 0.0035 & 0.31 & 0.0042 \\
Mn-PMN-PT & 240 & 0.0042 & 445 & 0.0070 & 0.50 & 0.0094 \\
\hline
\end{tabular}



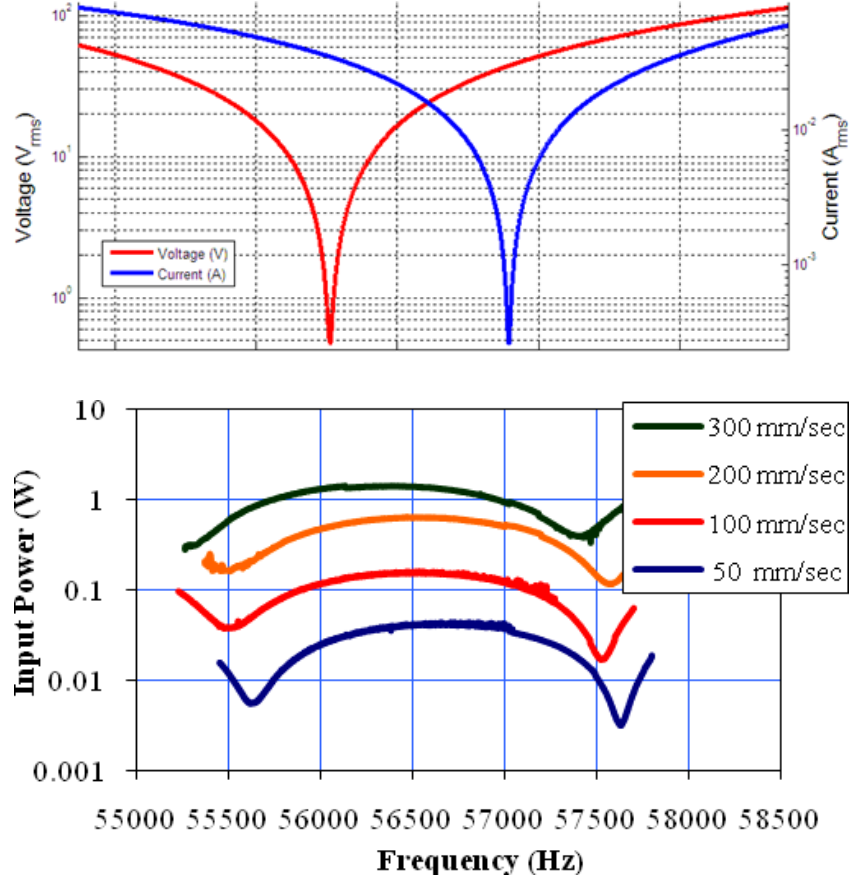

Fig. 10. (Color online) Frequency spectra of power under constant vibration velocity, conducted across the resonance and antiresonance frequencies (bottom). Controlled voltage and current are shown in top.

vibration velocity/amplitude conducted across the resonance and antiresonance frequencies. Note that the current and voltage are almost constant around the resonance and antiresonance frequencies, respectively. However, they are not perfectly constant to keep the vibration velocity/amplitude constant. It is obvious that the power required for generating the same tip vibration velocity of the rectangular plate is smaller for the antiresonance mode than for the resonance mode. This is the first experimental evidence of the higher efficiency at the antiresonance than that at the resonance.

Another attractive feature of HiPoCS Version V is the thermal analysis depicted in Fig. 11, which shows heat generation/distribution contours from the rectangular PZT8 plate driven under the same vibration velocity $(0.3 \mathrm{~m} / \mathrm{s})$ for the resonance (type A) and for the antiresonance (type B) mode. Note the key issue that heat generation is smaller for the antiresonance mode than it is for the resonance mode to generate the same vibration velocity, as supported by a higher $Q_{m}$ value.

\subsection{HiPoCS Version $V$ (Burst mode, 2010s)}

The resonance/antiresonance spectrum measurement mentioned in the previous section, is a continuous drive method, which inevitably is associated with temperature rise. This technique is suitable to the characterization of piezo-motors and



Fig. 11. (Color online) Heat generation from the rectangular PZT8 plate driven under the same vibration velocity (from 0.3 to $0.55 \mathrm{~m} / \mathrm{s}$ ) for the resonance (type A) mode and for the antiresonance (type B). 
piezo-transformers which are operated continuously. ${ }^{23,24}$ However, when we discuss the fundamental physical properties, we may need to exclude the temperature rise effect.

An alternative method which has the advantages of being quick exhibiting close-to-no heat rise is described in this section. The burst based characterization was accomplished by driving the piezoelectric at resonance for a given number of cycles and characterizing the ring-down behavior in one of two states; a traditional resonance condition ring-down under a short-circuit condition, ${ }^{25-28}$ and a new technique, which is introduced here for characterizing ring-down at antiresonance, i.e., under an open-circuit condition.

A rectangular plate piezoelectric sample was characterized using our HiPoCS Version 5 coupled with a specially designed circuit. The resonance mode characterization (i.e., short-circuit condition) does not require a special circuit, while for the antiresonance mode characterization, a high voltage blocking circuit is used to block current from flowing upstream to the amplifier when the ceramic transducer is left floating. The circuit used the TTL signal of the function generator, as the digital control of the blocking circuit for precise timing. Also for the antiresonance measurement a $500 \mathrm{X}$ differential voltage probe (Textronix P5205 $100 \mathrm{Mhz}$ High Voltage Differential Probe) was used to safely measure high voltages generated during an open-circuit condition.

A $80 \times 11 \times 2 \mathrm{~mm}^{3}$ plate sample of APC 841 was supported via two pins at the nodal point (center) and driven by a burst voltage at the first extensional $k_{31}$ resonance frequency initially. Then, the vibration ring-down performance was monitored after a sudden short-circuit or open-circuit condition.

Figure 12 plots the voltage, current and vibration velocity changes with time lapse after a burst resonance drive for short-circuit (top) and open-circuit (bottom) conditions.

The burst signal consists of an AC voltage with a frequency exactly equal to the resonance for only " $n$ " cycles $(n=10-100)$, and the signal package is repeated at a much lower frequency (typically $1-10 \mathrm{~Hz})$. As the AC signal is applied the piezoelectric ceramic gains motional current and vibration velocity. Once the driving signal is shut off, the build-up vibration starts to decay, which is called "ring-down". Notice that the ceramic is in a transient state and that the piezoelectric sample reaches maximum vibration amplitude only for one

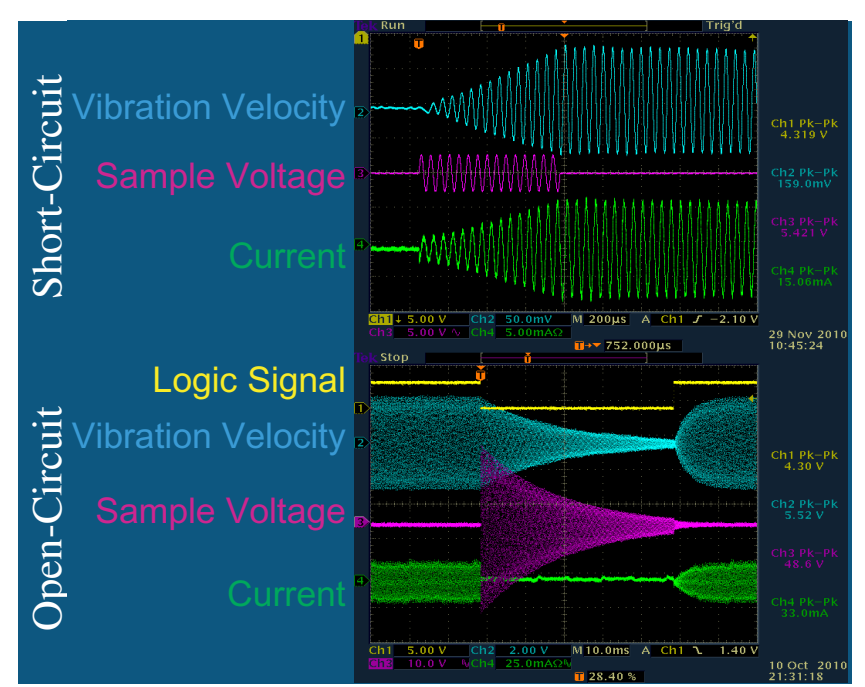

Fig. 12. (Color online) Voltage, current and vibration velocity changes with time lapse after a burst resonance drive for shortcircuit (top) and open-circuit (bottom) conditions.

single cycle. Though driving packets (bursts) are repeated, temperature rise in the ceramic can be avoided by utilization of a small duty ratio (long interval such as $0.1-1 \mathrm{~s}$ ), allowing loss evaluation in a thermal-effect-free condition. The applied burst interval is to be sufficient enough so as not to remain residual vibration from a prior burst package.

The short-circuit condition provides immediate zero voltage in the sample, and the ring-down of the vibration velocity and current reveal exactly the same trend. On the other hand, the open-circuit condition provides immediate zero current, and high voltage is generated due to a significant vibration (via direct piezoelectric effect). The ring-down performs exactly the same for the vibration velocity and voltage in this case.

The pseudo-exponential decay curves of the current $I$ and the vibration velocity $v$ (Fig. 13) are used to estimate the mechanical quality factor $Q_{A}$, while the voltage $V$ and the vibration velocity $v$ for the mechanical $Q_{B}$ estimation. For a sample with high quality factor the following equation holds;

$$
Q_{m}=\frac{\omega}{2(\alpha)}
$$

where

$$
\alpha=\frac{\ln \left(\frac{v_{t_{1}}}{v_{t_{2}}}\right)}{t_{1}-t_{2}} .
$$






Fig. 13. Determination of mechanical quality factor using ring-down characteristic of a resonant piezoelectric.

Here $I_{t 1}$ and $I_{t 2}$ are the amplitudes of the vibration velocity (or current for the $Q_{A}$, voltage for the $\left.Q_{B}\right)$ at time $t_{1}$ and $t_{2}$, respectively, shown in Fig. 13, and $\omega$ is angular velocity of the generated vibration velocity between $t_{1}$ and $t_{2}$. It is important to note that one cycle ring-down measurement can theoretically generate the vibration velocity dependence of the mechanical quality factors $Q_{A}$ or $Q_{B}$, by taking an infinitesimal time interval $\Delta t=t_{2}-t_{1}$.

Figure 14 depicted the ring-down current (resonance) and voltage (antiresonance) profiles,


putting the focus on the ringing frequency. The ringing frequency $20,460 \mathrm{~Hz}$ does not change from the initial drive to the ring-down period for the short-circuit condition, which is exactly the same as the resonance frequency. To the contrary, the initial resonance drive frequency $20,460 \mathrm{~Hz}$ suddenly changes to $21,430 \mathrm{~Hz}$ when the open-circuit condition is adopted. Note that this frequency is exactly the same as the antiresonance frequency of this sample.

Figure 15 summarized the resonance $Q_{A}$ and antiresonance $Q_{B}$ as a function of vibration velocity measured at $25^{\circ} \mathrm{C}$ under a burst mode, superposed with the $Q_{A}$ and $Q_{B}$ measured under a continuous (thermally-equilibrium) mode. Different from the previous data such as Figs. 9-11 (root-mean-square value of vibration velocity), this figure uses the unit of $(0-$ Peak $)$ value of vibration velocity $(\sqrt{2}$ times larger). We can conclude the following from Fig. 15:

(1) Mechanical quality factor $Q_{B}$ for antiresonance mode is larger than $Q_{A}$ for resonance mode in a whole vibration velocity range.

(2) Both $Q_{A}$ and $Q_{B}$ exhibit much larger values when a burst mode is used, in comparison with a continuous mode measurement. ${ }^{29,30}$ This

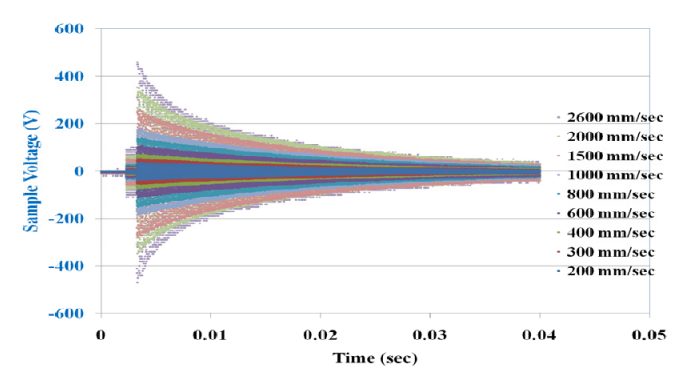

Antiresonance

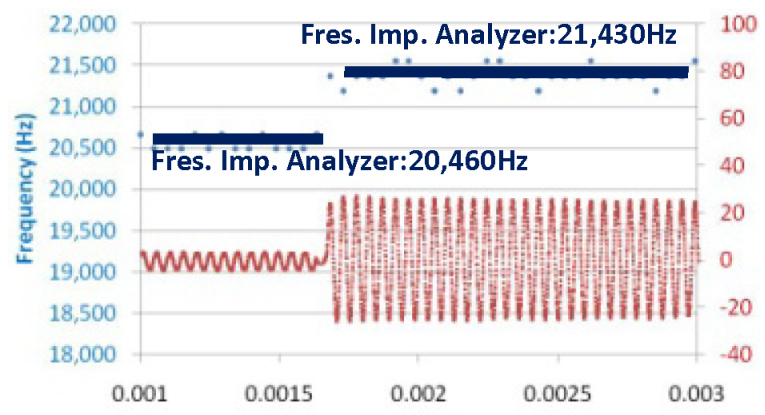

Fig. 14. (Color online) Ring-down current (resonance) and voltage (antiresonance) profiles, putting the focus on the ringing frequency. 


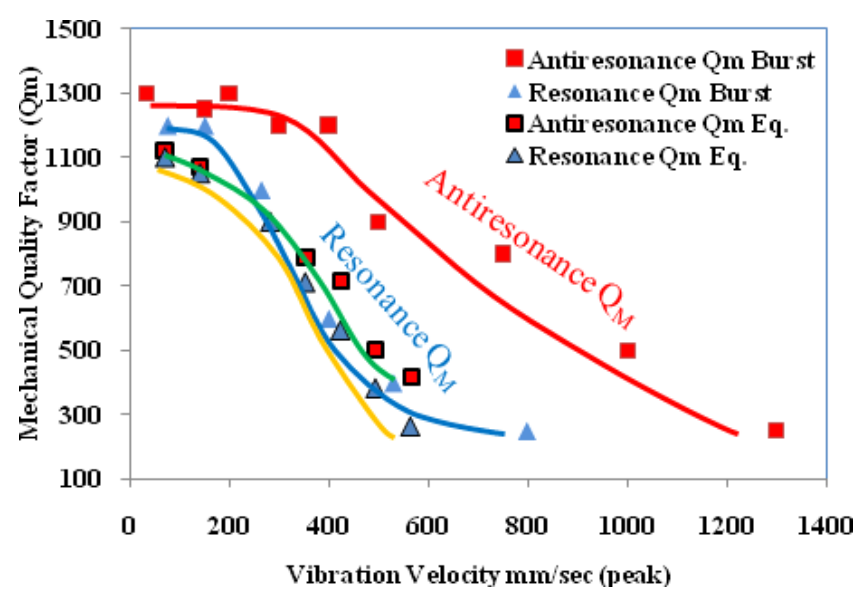

Fig. 15. (Color online) Resonance $Q_{A}$ and antiresonance $Q_{B}$ as a function of vibration velocity measured at $25^{\circ} \mathrm{C}$ under a burst mode, superposed with the $Q_{A}$ and $Q_{B}$ measured under a continuous (thermally-equilibrium) mode.

is explained by the temperature rise under a continuous mode.

\section{Loss Anisotropy}

We introduced a new loss phenomenology and innovative measuring methods based on the theory in this paper. First, quality factors at resonance and antiresonance for the $k_{31}, k_{33}, k_{t}$ and $k_{15}$ vibration modes are derived theoretically, and the methodology for determining loss factors in various orientations (i.e., loss anisotropy) is proposed. For simplicity, we focus on materials with $\infty \mathrm{mm}$ (equivalent to $6 \mathrm{~mm}$ ) crystal symmetry for deriving the loss factors of a polycrystalline ceramic, and 14 different loss factors among 20 in total can be obtained from the measurements. The mechanical quality factors for $k_{p}$ and $k_{31}^{\prime}$ are to be formulated in the future to complete the analysis. Second, we propose the experimental methods for measuring both mechanical quality factors $Q_{A}$ and $Q_{B}$ at the resonance and antiresonance modes: a continuous admittance/impedance spectrum measuring method (traditional with temperature rise) and a burst mode (to escape from the temperature effect). Results have shown that a piezoelectric driven in its antiresonance mode has significantly lower losses and higher quality factors than that in its resonance mode. Quality factors measured using the burst technique show higher magnitudes than that measured in equilibrium, indicating that thermal effect does significantly contribute to equilibrium condition characterization.
Table 2. Loss factors of APC 841 (unit: $\times 10^{-3}$ ) measured from $k_{31}$ and $k_{33}$ modes.

\begin{tabular}{lcccc}
\hline $\tan \phi_{11}^{\prime}$ & $\tan \phi_{33}^{\prime}$ & $\tan \delta_{33}^{\prime}$ & $\tan \theta_{31}^{\prime}$ & $\tan \theta_{33}^{\prime}$ \\
\hline 0.77 & 0.90 & 3.5 & 3.7 & 2.5 \\
$\tan \phi_{11}$ & $\tan \phi_{33}$ & $\tan \delta_{33}$ & $\tan \theta_{31}$ & $\tan \theta_{33}$ \\
0.44 & 0.54 & 3.1 & 0.25 & 1.5 \\
\hline
\end{tabular}

Finally, we show some example data for revealing the anisotropy of loss factors (unit: $\times 10^{-3}$ ) measured in APC 841 from $k_{31}$ and $k_{33}$ modes. Table 2 summarized the obtained dielectric, elastic and piezoelectric losses in various crystal orientations, which indicates:

(a) Piezoelectric (intensive) loss factors are not small as previously believed, but larger than the (intensive) dielectric and elastic losses: $\tan \theta^{\prime}>(1 / 2)\left(\tan \delta^{\prime}+\tan \phi^{\prime}\right)$.

(b) Intensive losses (with prime) are larger than the extensive (non-prime) losses.

(c) Loss anisotropy is obvious.

The hysteresis or loss is related primarily with domain re-orientation mechanism. However, mesoscopic domain models or physical meanings of piezoelectric losses need to wait to be developed from now on.

\section{Acknowledgement}

This study was supported by Office of Naval Research under the Grant Number: N00014-08-1-0912.

\section{References}

1. S. Cagatay, B. Koc, P. Moses and K. Uchino, Jpn. J. Appl. Phys. 43, 1429 (2004).

2. C. A. Rosen, Ceramic transformers and wave filters, in Proc. Electronic Component Symp. (1957), pp. $205-211$.

3. K. Uchino, H. Negishi and T. Hirose, Jpn. J. Appl. Phys. 28, 47 (1989).

4. N. Aurelle, D. Guyomar, C. Richard, P. Gonnard and L. Eyraud, Ultrasonics 34, 187 (1996).

5. S. Priya, D. Viehland, A. V. Carazo, J. Ryu and K. Uchino, J. Appl. Phys. 90, 1469 (2001).

6. G. E. Martin, in Proc. Ultrasonics Symp. (1974), pp. $613-617$.

7. T. Ikeda, Fundamentals of Piezoelectric Materials Science (Ohmsha, Tokyo) (1984), p. 83. 
8. J. Zheng, S. Takahashi, S. Yoshikawa, K. Uchino and J. W. C. de Vries, J. Amer. Ceram. Soc. 79, 3193 (1996).

9. K. Uchino, J. H. Zheng, Y. H. Chen, X. H. Du, J. Ryu, Y. Gao and S. Ural, J. Mater. Sci. 41, 217 (2006).

10. S. O. Ural, S. Tuncdemir, Y. Zhuang and K. Uchino, Jpn. J. Appl. Phys. 48, 056509 (2009).

11. K. Uchino and S. Hirose, IEEE Trans. Ultrason. Ferroelectr. Freq. Control 48, 307 (2001).

12. S. Hirose, M. Aoyagi, Y. Tomikawa, S. Takahashi and K. Uchino, Ultrasonics 34, 213 (1996).

13. A. V. Mezheritsky, IEEE Trans. Ultrason. Ferroelectr. Freq. Control 49, 484 (2002).

14. IRE Standards on Piezoelectric Crystals: Measurements of Piezoelectric Ceramics (1961), p. 1163; IEEE Std. 176 (1987), p. 54.

15. G. Arlt and H. Dederichs, Ferroelectrics 29, 47 (1980).

16. K. Uchino and J. R. Giniewicz, Micromechatronics (CRC Press, NY, 2003).

17. K. Uchino, J. Zheng, A. Joshi, Y. H. Chen, S. Yoshikawa, S. Hirose, S. Takahashi and J. W. D. de Vries, J. Electroceram. 2, 33 (1998).

18. Y. Zhuang, S. O. Ural, A. Rajapurkar, S. Tuncdemir, A. Amin and K. Uchino, Jpn. J. Appl. Phys. 48, 041401 (2009).
19. Y. Zhuang, S. O. Ural, S. Tuncdemir, A. Amin and K. Uchino, Jpn. J. Appl. Phys. 49021503 (2010).

20. W. Cao, S. Zhu and B. Jiang, J. Appl. Phys. 83, 4415 (1998).

21. S. O. Ural, S. Tuncdemir, Y. Zhuang and K. Uchino, Jpn. J. Appl. Phys. 48, 056509 (2009).

22. A. Rajapurkar, S. O. Ural, H.-Y. Lee, A. Amin and K. Uchino, Jpn. J. Appl. Phys. 49, 071502 (2010).

23. K. Uchino, Piezoelectric Actuators and Ultrasonic Motors, ed. H. L. Tuller (Kluwer Acad. Pub., Norwell, MA, 1997).

24. S. Priya, D. Viehland, A. Vazquez Carazo, J. Ryu and K. Uchino, J. Appl. Phys. 90, 1469 (2001).

25. M. Umeda, K. Nakamura and S. Ueha, Jpn. J. Appl. Phys. 37, 5322 (1998).

26. M. Nishihira, M. Kaido and K. Imano, Jpn. J. Appl. Phys. 41, 3354 (2002).

27. K. Imano and D. Okuyama, Trans. IEICE J74-A, 797 (1991).

28. A. Albareda, R. Perez, J. A. Casals, J. E. Garcia and D. A. Ochoa, Trans. UFFC 54, 2175 (2007).

29. S. Takahashi, Y. Sasaki, M. Umeda, K. Nakamura and S. Ueha, in Proc. IEEE Int. Symp. Appl. Ferroelectr., (2001), p. 11.

30. Y. Hiruma, T. Watanabe, H. Nagata and T. Takenaka, Jpn. J. Appl. Phys. 47, 7659 (2008). 